\title{
NOTA \\ PRESENCIA DE POBLACIONES NATURALIZADAS DE RANA TORO (Lithobates catesbeianus) EN LA ZONA DE AMORTIGUAMIENTO ORIENTAL DEL PARQUE NACIONAL IGUAZÚ, ARGENTINA
}

\author{
Carlos Ariel López ${ }^{1 *}$ \& Emanuel Grassi²
}

${ }^{1}$ Instituto Nacional de Medicina Tropical, Almafuerte y Ambar s/n, Puerto Iguazú, Misiones.

2 Instituto Misionero de Biodiversidad, El Dorado y Ruta Nac. 12 Puerto Iguazú, Misiones.

*Autor para correspondencia: lopez_arielc@yahoo.com.ar

\section{RESUMEN}

Se presentan datos sobre las poblaciones naturalizadas de rana toro (Lithobates catesbeianus) registradas en la zona de amortiguamiento del Parque Nacional Iguazú. Este hallazgo pone de manifiesto la necesidad de medidas de control y monitoreo de las actividades productivas en la periferia del área protegida más biodiversa del país.

Palabras clave: rana toro, Parque Nacional Iguazú, invasión biológica, Selva Atlántica

\begin{abstract}
Presence of naturalized populations of bullfrog (Lithobates catesbeianus) in the eastern buffer zone of Iguazú National Park, Argentina. We present data about the naturalized populations of the bullfrog (Lithobates catesbeianus) recorded in the buffer zone of the Iguazú National Park. This finding highlights the need of control and monitoring measures of productive activities in the periphery of the country's most biodiverse protected area.
\end{abstract}

Keywords: bullfrog, Iguazú National Park, biological invasion, Atlantic Rainforest

Las poblaciones de anfibios están en riesgo a nivel mundial y una de las principales amenazas es la invasión de especies exóticas y los agentes patógenos asociados (Bucciarelli et al., 2014; Epstein \& Storfer 2016). La rana toro americana (Lithobates catesbeianus), está considerada entre las 100 especies invasoras más peligrosas del mundo (Lowe et al., 2000). Los impactos ecológicos como especie invasora varían de acuerdo a la etapa de su ciclo de vida; las larvas alcanzan gran tamaño -superan los $15 \mathrm{~cm}$. de longitud- y muestran gran plasticidad trófica, pudiendo alterar las poblaciones de la microflora de los ambientes acuáticos, alimentarse de las larvas de las especies autóctonas de anfibios o alterar su comportamiento (Boone et al., 2004; Arellano et al., 2009), mientras que el adulto alcanza los 20 centímetros de longitud de hocico-cloaca y supera los 800 gramos de peso (GISD, 2018) siendo un potencial predador de un amplio rango de pequeños vertebrados, incluyendo el canibalismo (Wu et al., 2005; Leivas et al.,2013; Silva et al., 2011, 2016; Laufer \& Gobel, 2017). Puede interferir el comportamiento y el nicho acústico de anfibios nativos (Forti et al., 2017) así como actuar como vector de patógenos como ranavirus y el hongo Batrachochytrium dendrobatidis $(B d)$, identificado como responsable de la mortandad de poblaciones de anfibios en ambientes alterados y prístinos del mundo (Daszak et al., 2003; Skerrat et al., 2007; Both \& Grant, 2012; Greenspan et al., 2012; Rodder et al., 2013; Price et al., 2014). Desde el año 2000 la rana toro se ha encontrado en nueve países de América Latina (Frost, 2018).

La primera notificación de su presencia en la naturaleza en Argentina fue en la provincia de San Juan (Sanabria et al., 2005), posteriormente se registró en la provincias de Misiones (Pereyra et al., 2006), Buenos Aires (Barrasso et al., 2009), Córdoba (Akmentins et al., 2009) y Salta (Akmentins \& Cardozo, 2009). Los trabajos de campo siguen aportando nuevos registros de poblaciones silvestres en esas provincias (Sanabria et al., 2011), poniendo en evidencia que la distribución actual de la especie en el país es aún desconocida (Walter Prado com. pers.) no siendo posible caracterizar la etapa de invasión en los términos de Blackburn et al. (2011). Hasta ahora ningún área protegida en el país estaba amenazada por la presencia de la rana exótica. Especial atención merece la presencia del hongo $B d$ en poblaciones naturales de anfibios, el primer caso de chytridiomycosis en Argentina fue reportado por Herrera et al. (2005), mientras que Arellano et al. (2009) informaron el hallazgo de un individuo de Elachistocleis bicolor infectado por el hongo en la provincia de Misiones, donde se encontrarían zonas con aptitud para el desarrollo de $B d$ en el centro y extremo norte la provincia (Ghirardi et al., 2011). Hasta la fecha se han encontrado unas 20 especies de anfibios nativos salvajes infectados con $B d$ y ya se han reportado poblaciones naturalizadas de rana toro actuando como vectores del hongo en el oeste del país, aunque todavía no se puede establecer una relación causal y la descripción de los mecanismos de dispersión fúngica (Ghirardi et al., 2017).

El 25 de agosto de 2017, a las 10:00 a.m., se grabó un individuo de rana toro vocalizando en un 
pequeño cuerpo de agua (25.70608 $\left.\mathrm{S}-54.1415^{\circ} \mathrm{W}\right)$, ubicado en una chacra dentro de la zona de amortiguamiento del Parque Nacional Iguazú (PNI) (Fig. 1A), a sólo 550 metros de su límite. En dicho cuerpo de agua se verificó la presencia de larvas y especímenes post-metamórficos de la especie. En las observaciones nocturnas se registró fotográficamente la presencia de una hembra adulta y un macho vocalizando. Otras especies de anuros se observaron vocalizando en el mismo cuerpo de agua fueron: Dendropsophus minutus desde la vegetación herbácea emergente, Rhinella diptycha sobre un tronco flotante y desde los márgenes, Physalaemus cuvieri en pequeños cuerpos de agua, de unos pocos centímetros de profundidad asociados al cuerpo principal, o en pisadas inundadas de ganado, mientras que Leptodactylus fuscus vocalizaba en las márgenes, debajo de troncos o rocas. También se observaron larvas de especies nativas en áreas poco profundas del cuerpo de agua, entre las algas.

El cuerpo de agua es un manantial de régimen permanente, de forma subcircular y márgenes irregulares - 10 metros de largo por 6 metros de ancho con una profundidad aproximada máxima de un metro en el centro-, el agua es limpia y el lecho es arcilloarenoso. El mismo presenta vegetación emergente en las márgenes sombreadas y vegetación flotante (Familia Nymphaeaceae), con poca superficie libre, y abundantes algas sumergidas. Se encuentra ubicado en un potrero típico de los que se observan en las propiedades de la zona de amortiguamiento este del PNI, constituyendo una práctica común la construcción de embalses para la provisión de agua para el ganado y el autoconsumo.

Fueron colectados siete renacuajos -media de la longitud total $(L T)=10.1 \pm 0.3 \mathrm{~cm}$, rango $L T=8.7-11.1$ cm., $\mathrm{n}=7-\mathrm{y}$ llevados al laboratorio donde, luego de entre 112 y 140 días, completaron la metamorfosis (Figura 1B) -media de la longitud hocico-cloaca $=4.40$ \pm 0.07 , rango $\mathrm{LHC}=4.2-4.5 \mathrm{~cm}$., $\mathrm{n}=4$ - (número de colección: INMET 080-84). Uno de estos renacuajos fue encontrado moribundo, con manchas rojizas en el abdomen, si bien un análisis preliminar -tinción y observación del tejido de la zona con microscopio

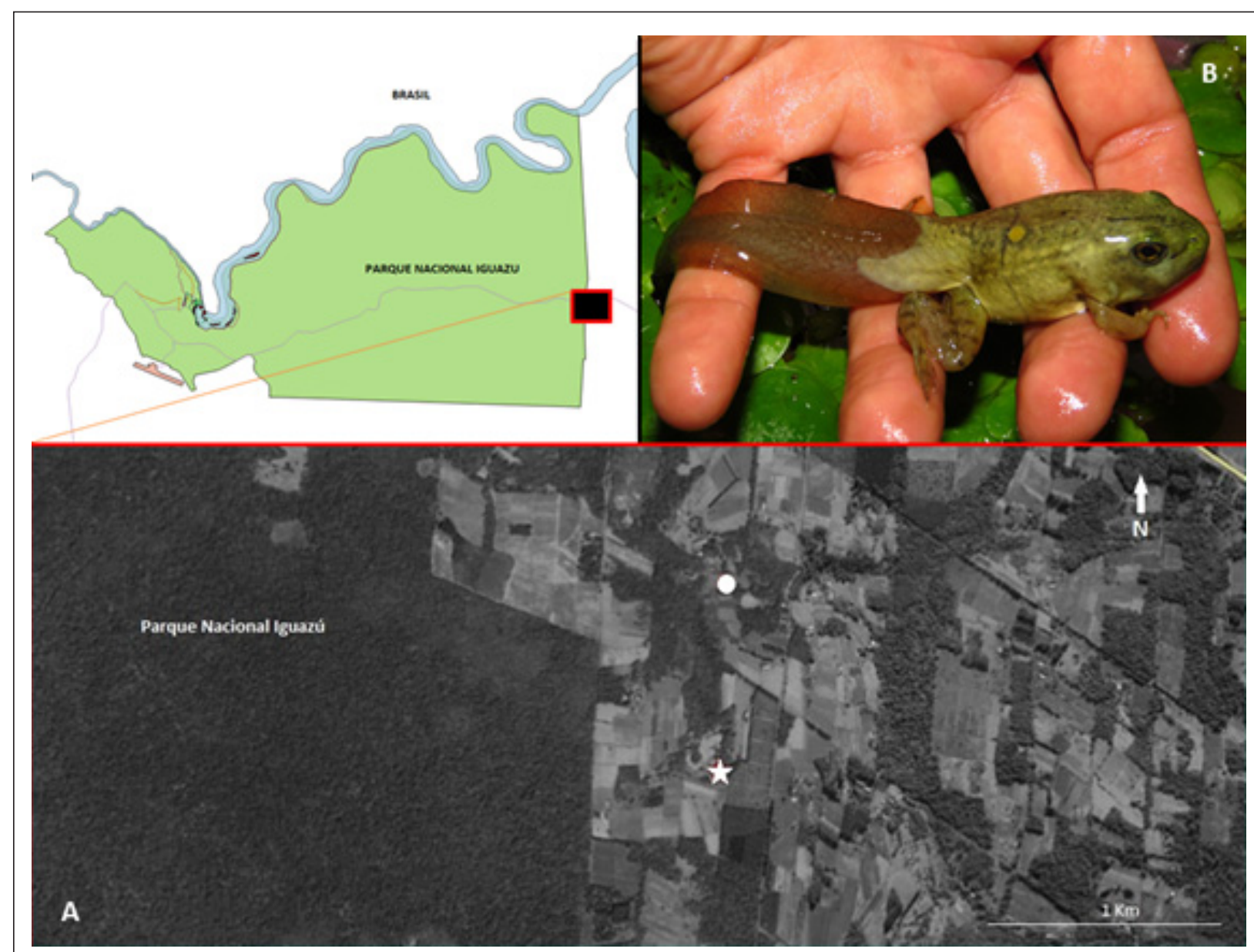

Fig. 1. A) Ubicación del registro de Rana catesbeiana (estrella) en la zona de amortiguamiento del PNI, y del posible origen de la invasión (circulo). B) Larva colectada completando la metamorfosis en el laboratorio. 
óptico- no evidenció la presencia de zoosporas de $B d$, se requerirá de técnicas moleculares para el seguimiento de la presencia del hongo en la población exótica.

En entrevistas informales los vecinos relataron que a menos de un kilómetro (25.69912º S - 54.14017W) del sitio del hallazgo, en el arroyo Carabosá, funcionó un criadero no registrado de la especie hasta el año 2006. En este lugar el arroyo fue modificado para lograr un embalse de 200 metros de largo por 80 metros de ancho donde se construyeron las instalaciones de cría que, luego de intensas lluvias, se inundaron y los individuos escaparon, constituyendo quizás el punto de origen de esta invasión. Una inspección durante el día permitió observar cerca de una docena de larvas de rana toro en zonas de baja profundidad del cuerpo de agua. El arroyo Carabosá es tributario del Arroyo Yacuí, que discurre dentro del PNI hasta su desembocadura en el Rio Iguazú, pudiendo funcionar como una vía de acceso al área protegida.

Los modelos de distribución de especies anticiparon que la región sur de la Mata Atlántica o Selva Atlántica exhibe condiciones adecuadas para la invasión de la rana toro (Nori et al., 2011a; Ficetola et al., 2007; Giovanelli et al., 2007), recientemente Barbosa et al. (2017) y Loyola et al. (2012) comunicaron que las áreas protegidas de la misma región presentan aptitud climática para la especie. Actualmente, el sureste de Brasil y el noreste de Argentina están experimentando la mayor invasión de rana toro de Sudamérica (Both et al., 2011, Nori et al., 2011b), esta situación representa un riesgo para las especies de anfibios en áreas poco estudiadas de la ecorregión (Vancine et al., 2018) y, especialmente, en la provincia de Misiones y el PNI que cuenta con un ensamble de anuros nativos de unas 32 especies (Ariel López en preparación).

Por lo que sabemos, esta es la población de rana toro más septentrional del país y la primera que amenaza a un área protegida de jurisdicción nacional. Estas poblaciones no estarían vinculas a las provenientes del sur de Brasil (Pereyra et al., 2006) que están invadiendo el este de la provincia, siendo consecuencia de la actividad productiva local en la zona de amortiguamiento del PNI. Este hallazgo pone en evidencia la necesidad de reglamentar y monitorear las actividades productivas en la zona de amortiguación de las áreas protegidas nacionales (St Amour et al., 2008; Madalozzo et al., 2016), en particular del $\mathrm{PNI}$, el área protegida más biodiversa del país.

\section{AGRADECIMIENTOS}

Agradecemos a las familias Bioloso y Fiebke por su gentileza, a Victoria Gnaso, Nilso Molina, Nadia Graf y Alejandro Cárdenas por la asistencia en el campo.

\section{REFERENCIAS}

Akmentins, M.S. \& Cardozo D.E. 2009. American bullfrog Rana catesbeiana (Shaw, 1802) invasion in Argentina. Biological Invasions, 12(4): 735-737.

Akmentins M.S., Pereyra L.C. \& Lescano J.N. 2009. Primer registro de una población asilvestrada de rana toro (Rana catesbeiana) en la provincia de Córdoba, Argentina, notas sobre la biología de la especie. Cuadernos de Herpetología, 23(1): 25-32.

Arellano M.L., Natale G.S., Grilli P.G., Barrasso D.A., Steciow M.M. \& Lavilla E.O. 2017. Hostpathogen relationships between the chytrid fungus Batrachochytrium dendrobatidis and tadpoles of five South American anuran species. Herpetological Journal, 27: 33-39.

Barbosa F.G., Both C. \& Araùjo M.B. 2017. Invasive American bullfrog and African Clawed Frogs in South America High Suitability of Occurrence in Biodiversity Hotspots. Zoological Studies, 56: 28.

Barrasso D.A., Cajade R., Nenda, S.J., Baloriani G. \& Herrera R. 2009. Introduction of the American Bullfrog Lithobates catesbeianus (Anura: Ranidae) in natural and modified environments: an increasing conservation problem in Argentina. South American Journal of Herpetology 4 (1): 69- 75.

Boone M.D., Little E.E. \& Semlitsch R.D. 2004. Overwintered Bullfrog Tadpoles Negatively Affect Salamanders and Anurans in Native Amphibian Communities. Copeia, 2004(3): 683-690.

Both C. \& Grant T. 2012. Biological invasions and the acoustic niche: the effect of bullfrog calls on the acoustic signals of white-banded tree frogs. Biology Letters, 8(5): 714-716.

Both C., Lingnau R., Santos-Jr. A., Madalozzo B., Pedrosa Lima L. \& Grant T. 2011. Widespread occurrence of the American Bullfrog, Lithobates catesbeianus (SHAW, 1802) (ANURA: RANIDAE), in Brazil. South American Journal of Herpetology, 6(2): 127-134.

Blackburn T.M., Pysek P., Bacher S., Carlton J.T., Duncan R.P., Jarosík V., Wilson J.R.U. \& Richardson D.M. 2011. A proposed unified framework for biological invasions. Trends in Ecology \& Evolution, 26(7): 333- 339.

Bucciarelli G.M., Blaustein A.R., Garcia T.S. \& KatsL.B. 2014. Invasion Complexities: The Diverse Impacts of Nonnative Species on Amphibians. Copeia, 2014(4): 611-632.

Daszak P., Cunningham A.A. \& Hyatt A.D. 2003. Infectious disease and Amphibian population declines. Diversity and Distribution 9: 141-150.

Epstein B. \& Storfer A. 2016. Comparative Genomics of an Emerging Amphibian Virus. Genes Genomes Genetics, 6(1): 15-27. 
Ficetola G.F., Thuiller W. \& Miaud C. 2007. Prediction and validation of the potential global distribution of a problematic alien species - the American bullfrog. Diversity and Distribution, 13: 476485.

Forti L.R., Becker C.G., Tacioli L., Pereira V.R., Santos A.C.F.A., Oliveira I., Haddad C.F.B. \& Toledo L.F. 2017. Perspectives on invasive amphibians in Brazil. PLoS ONE, 12(9): e0184703.

Frost D.R. 2018. Amphibian Specie of the World: an Online Reference, Version 6.0 (access 5 july). Electronic Database accessible at Http:// research .amnh.org//herpetology/amphibian/ index.htlm. American Museum of Natural History, New York, USA.

Ghirardi R., López J.A., Sanabria E.A., Quiroga L.B. \& Levy M.G. 2017. Pathogenic fungus in naturalizada populations of the invasive North American bullfrog in Argentina. Belgian Journal of Zoology, 147(2): 81-86.

Ghirardi R., López J.A., Scarabotti P.A., Steciow M.M. \& Perotti M.G. 2011. First record of the chytrid fungus in Lithobates catesbeianus from Argentina: exotic species and conservation. Revista Mexicana de Biodiversidad, 82: 13371339.

Giovanelli J.G.R., Haddad C.F.B. \& Alexandrino J. 2007. Predicting the potential distribution of the alien invasive American bullfrog (Rana catesbeiana) in Brazil. Biological Invasions, 10(5): 585-590.

GISD 2018. Global Invasive Species Database (2018) Species profile: Rana catesbeiana. Downloaded from http://www.iucngisd.org/gisd/ species.php?sc=80 on 29-06-2018.

Greenspan S.E., Calhoun A.J.K., Longcore J.E. \& Levy M.G. 2012. Transmission of Batrachochytrium dendrobatidis to wood frogs (Lithobates sylvaticus) via a bullfrog ( $L$. catesbeianus) vector. Journal of Wildlife Diseases, 48(3): 575-582.

Herrera R.A., Steciow M.M. \& Natale G.S. 2005. Chytrid fungus parasitizing the wild amphibian Leptodactylus ocellatus (Anura: Leptodactylidae) in Argentina. Diseases of Aquatic Organisms, 64: 247-252.

Laufer G. \& Gobel N. 2017. Habitat degradation and biological invasions as a cause of amphibian richness loss: a case report in Aceguá, Cerro Largo, Uruguay. Phyllomedusa, 16(2):289293.

Leivas P.T., Savaris M., Lampert S. \& Lucas E.M. 2013. Predation of Odontophrynus americanus (Anura: Odontophrynidae) by the Invasive Species Lithobates catesbeianus (Anura: Ranidae) in an Araucaria Forest Remnant in Southern Brazil. Herpetology Notes, 6(1): 6036.
Lowe S., Browne N., Boudpelas S. \& Poorter M. 2000. 100 of the World's Worst Invasive Alien Species A selection from the Global Invasive Species Database. Published by the Invasive Species Group (ISSG) a specialist group of the Species Survival Comission (SSC) of the World Conservation Union (IUCN), $12 \mathrm{pp}$. First published as special liftout in Aliens 12 December 2000. Updated and reprinted version November 2004.

Loyola R.D., Nabout J.C., Trindade-Filho J., Lemes P., Urbina-Cardona J.N., Dobrovolski R., Sagnori M.D. \& Diniz-Filho J.A.F. 2012. Climate Change Might Drive Species into Reserves: A Case Study of the American Bullfrog in the Atlantic Forest Biodiversity Hotspot. Alytes, 29(1-4): 6174.

Madalozzo B., Both C. \& Cechin S. 2016. Can Protected Areas with Agricultural Edges Avoid Invasions? The Case of Bullfrogs in the Southern Atlantic Rainforest in Brazil. Zoological Studies, 55(51): 1-13.

Nori J., Akmentins M.S., Ghirardi R., Frutos N. \& Leynaud G.C. 2011b. American bullfrog invasion in Argentina: where should we take urgent measures? Biodiversity Conservation, 20(5): 1125-1132.

Nori J., Urbina-Cardona J.N., Loyola R.D., Lescano J.N. \& Leynaud G.C. 2011a. Climate Change and American Bullfrog Invasion: What Could We Expect in South America? PLoS ONE, 6(10): e25718. doi:10.1371/journal.pone.0025718.

Pereyra M.O., Baldo D. \& Krausuk E.R. 2006. La "rana toro" en la Selva Atlántica Interior de Argentina: Un nuevo problema de conservación. Cuadernos de Herpetología, 20(1): 37-40.

Price S.J., Garner T.W.J., Nichols R.A., Balloux F., Ayres C., de Alba A.M.C. \& Bosch J. 2014. Collapse of Amphibian Communities Due to an Introduced Ranavirus. Current Biology, 24: 25862591.

Rödder D., Schulte U. \& Toledo L.F. 2013. High Environmental Niche Overlap between the Fungus Batrachochytrium dendrobatidis and Invasive Bullfrogs (Lithobates catesbeianus) Enhance the Potential of Disease Transmission in the Americas. North-Western Journal of Zoology, 9(1): 178-84.

Sanabria E.A., Quiroga L.B. \& Acosta J.C. 2005. Dieta de Leptodactylus ocellatus (Linnaeus, 1758) (Anura: Leptodactylidae) en un humedal del oeste de Argentina. Revista Peruana de Biología, 12(3): 472- 477.

Sanabria E., Ripoll Y., Jordan M., Quiroga L., Ariza M., Guillemain M.,Pérez M. \& Chávez H. 2011. A new record for American Bullfrog (Lithobates catesbeianus) in San Juan, Argentina. Revista Mexicana de Biodiversidad, 82: 311-313. 
Silva E.T., Ribeiro Filho O.P. \& Feio R.N. 2011. Predation of native anurans by invasive bullfrogs in southeastern Brazil: spatial variation and effect of microhabitat use by prey. South American Journal of Herpetology, 6(1): 110.

Silva E.T., Ribeiro Filho O.P. \& Feio R.N. 2016. Food Habits of Invasive Bullfrogs and Native ThinToed Frogs Occurring in Sympatry in Southeastern Brazil. South American Journal of Herpetology, 11(1): 25-33.

Skerrat L.F., Berger L., Speare R., Cashing S., McDonald K.R., Phillott A.D., Hines H.B. \& Kenyon N. 2007. Spread of Chitridiomycosis has caused the rapid global decline and extinction of frogs. EcoHealth 4: 25.

St-Amour V., Wong W.M., Garner T.W.J. \& Lesbarrères D. 2008. Anthropogenic Influence on Prevalence of 2 Amphibian Pathogens. Emerging Infectious Diseases, 14 (7): 1175-1176.
Vancine M., da Silva Duarte K., de Souza Y.S., Ribeiro Giovanelli J.G., Martins-Sobrinho P.M., López A., Parelli Bovo R., Maffei F., Bruzzi Lion M., Ribeiro Júnior J. W., Brassaloti R., Rocha da Costa C.O., Oliveira Sawakuchi H., Rodriguez Forti L., Cacciali P., Bertoluci J., Baptista Haddad C.F. \& Ribeiro M.C. 2018. ATLANTIC AMPHIBIANS: a data set of amphibian communities from the Atlantic Forests of South America. Ecology, 99(7): 1692.

Wu Z., Li Y., Wang Y. \&. Adams M.J 2005. Diet of Introduced Bullfrogs (Rana catesbeiana): Predation on and Diet Overlap with Native Frogs on Daishan Island, China. Journal of Herpetology, 39(4):668-74.

Fecha de Recepción: 28 de enero de 2019 Fecha de Aceptación: 07 de noviembre de 2019 